\title{
Clinicopathological Features and Metastatic Pattern of Hepatocellular Carcinoma: An Autopsy Study of 398 Patients
}

\author{
Manuel Schlageter Luca Quagliata Matthias Matter Valeria Perrina \\ Luigi Tornillo Luigi Terracciano \\ Department of Pathology, University Hospital Basel, Basel, Switzerland
}

\section{Key Words}

Autopsy · Hepatocellular carcinoma - Metastasis .

Epidemiology · Pathology

\begin{abstract}
Objectives: Analysis of a large local autopsy collective to gather epidemiological and histopathological data on hepatocellular carcinoma (HCC). Methods: We examined a large dataset of 44,104 autopsies performed at the Institute of $\mathrm{Pa}$ thology, Basel, Switzerland, including 2 autopsy collectives (1969-1983 and 1988-2012) to gather current data on HCC in the advanced stage. A total of 398 HCC were diagnosed, accounting for around 1\% of all autopsies. Results: As expected, most patients developing $\mathrm{HCC}$ had advanced stages of liver fibrosis or cirrhosis (F3/F4). However, in the more recent autopsy collective (1988-2012), our data also show an increase of HCC arising in livers without or with only mild to moderate fibrosis (F0-F2). Extrahepatic metastasis was found in 156 of 398 HCC (39.1\%), with lung metastasis (74.5\%) being the most common, followed by the bones (24.8\%) and adrenal glands (19.1\%). Conclusions: Our data therefore seem to suggest that, in the last 2 decades, despite the introduction of new therapeutic modalities for HCC, no significant changes have been observed regarding the metastatic pattern of advanced HCC.

๑) 2016 S. Karger AG, Basel
\end{abstract}

\section{Introduction}

Hepatocellular carcinoma (HCC) is the third leading cause of cancer-related death worldwide and a leading cause of mortality among cirrhotic patients [1]. About $80 \%$ of HCC is due to hepatitis B virus and/or hepatitis C virus infections [2]. However, recent studies show an increasing shift to HCCs due to alcoholic/nonalcoholic steatohepatitis [3]. Most HCCs develop in cirrhotic livers, but a small percentage develops in noncirrhotic liver tissue. This subgroup is particularly interesting, since the pathogenesis of these carcinomas most likely differs from common risk factors such as viral hepatitis, alcoholic and/or nonalcoholic steatohepatitis and aflatoxin exposure [4]. Classic symptoms are usually associated with the underlying cirrhosis (i.e. jaundice, ascites and loss of appetite) and, rarely, pain in the upper right quadrant is reported. Unlike most malignancies, the incidence of liver cancer and mortality in the general population has increased significantly in the past 20 years. Furthermore, epidemiologic evidence indicates that it will still increase significantly in several Western countries such as the USA during the next decades $[5,6]$. In particular, recent data regarding the analysis of mortality from HCC in Europe indicate a large variability across European countries, with upward trends in Austria, Germany, Switzer-

\section{KARGER}

E-Mail karger@karger.com

www.karger.com/pat
(C) 2016 S. Karger AG, Basel

1015-2008/16/0836-0301\$39.50/0
Manuel Schlageter, MD

Department of Pathology, University Hospital Basel

Schönbeinstrasse 40

$\mathrm{CH}-4031$ Basel (Switzerland)

E-Mail Manueleric.schlageter@usb.ch 


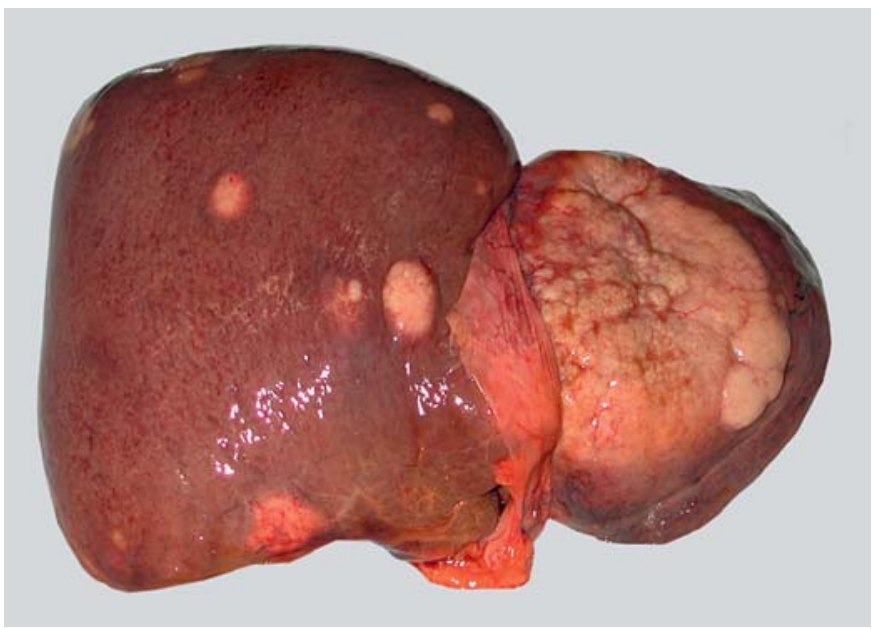

Fig. 1. Macroscopy of a multinodular, bilobal HCC in a non-fibrotic liver specimen.

land and other northern countries in general, but significant declines in recent years in a few countries such as France and Italy [7].

According to our literature review, there are no recent studies examining the epidemiology and the metastatic patterns of HCC in an autoptic series. The most recent studies examining the epidemiology and metastatic patterns of HCC in an autoptic collective date back to the early 1990s $[8,9]$. Given the crucial role of detection of preoperative metastases for treatment selection, a better understanding of the metastatic behavior of HCC is highly desirable. Furthermore, in the last decades, several etiologic and clinical features regarding HCC have changed, leading to a new scenario of HCC from a clinical point of view.

This study is therefore mainly aimed at determining the patterns of the metastatic spread of HCC and evaluating whether, in the last decades, important changes regarding risk factors and clinical features have also modified the clinicopathological scenario of HCC at the autoptic level.

\section{Patients and Methods}

\section{Study Groups}

In a consecutive series of 44,104 autopsies performed at the Institute of Pathology of University Hospital Basel between 1969 and 1983 and 1988 and 2012, we identified 398 cases of patients with HCC. The databases were electronically searched and only the cases diagnosed with HCC were evaluated. We analyzed the 2 corre-
Table 1. Patient demographics

\begin{tabular}{|c|c|c|c|}
\hline & $\begin{array}{l}1988-2012 \\
(\mathrm{n}=158)\end{array}$ & $\begin{array}{l}1969-1983 \\
(\mathrm{n}=240)\end{array}$ & $\begin{array}{l}\mathrm{p} \\
\text { value }\end{array}$ \\
\hline Male gender & 77 & 78 & n.s. \\
\hline Mean age, years & $72 \pm 11$ & $72 \pm 10$ & n.s. \\
\hline \multicolumn{4}{|l|}{ Underlying disease } \\
\hline None & 2.5 & 0 & n.s. \\
\hline Alcohol & 69.7 & 66.3 & n.s. \\
\hline Viral hepatitis & 29.5 & 36.6 & n.s. \\
\hline NASH/ASH & 1.6 & 0 & n.s. \\
\hline Hemochromatosis & 2.5 & 1.7 & n.s. \\
\hline Biliary & 1.6 & 0.6 & n.s. \\
\hline a1-antitrypsin deficiency & 2.5 & 0.6 & n.s. \\
\hline \multicolumn{4}{|l|}{ Fibrosis $^{1}$} \\
\hline F0 & 1.3 & 0.4 & n.s. \\
\hline $\mathrm{F} 1$ & 7.6 & 1.3 & $<0.05$ \\
\hline $\mathrm{F} 2$ & 12 & 1.3 & $<0.05$ \\
\hline $\mathrm{F} 3$ & 8.9 & 6.7 & n.s. \\
\hline F4 & 69 & 77.5 & n.s. \\
\hline Not evaluable & 1.3 & 12.9 & $<0.05$ \\
\hline \multicolumn{4}{|l|}{ Inflammatory activity ${ }^{1}$} \\
\hline A0 & 23.4 & 4.5 & $<0.05$ \\
\hline A1 & 31 & 63.6 & $<0.05$ \\
\hline $\mathrm{A} 2$ & 24.7 & 22.7 & n.s. \\
\hline A3 & 3.2 & 9.1 & n.s. \\
\hline Not evaluable & 17 & 0 & \\
\hline \multicolumn{4}{|l|}{ Steatosis } \\
\hline $0-10 \%$ & 75 & n.a. & n.a. \\
\hline $11-33 \%$ & 14.6 & n.a. & n.a. \\
\hline $34-100 \%$ & 10.4 & n.a. & n.a. \\
\hline Ballooning present & 2.3 & n.a. & n.a. \\
\hline
\end{tabular}

Values denote the percentage of patients, unless otherwise indicated. NASH/ASH = Non-alcoholic steatohepatitis/alcoholic steatohepatitis; n.s. = not significant; n.a. = not available.

${ }^{1}$ Measured according to the METAVIR scoring system.

sponding databases for autopsy patients diagnosed with HCC and created 2 separate collectives.

\section{8-2012 Collective}

In the more recent database on 1988-2012, we found 158 HCC out of 15,104 autopsy cases. We reviewed the autopsy reports and the clinical information from the clinical database of our hospital. Histology slides were reevaluated for different histological parameters (table 1) in order to confirm the diagnosis and to characterize the neoplasia according to current standards. Tumor stage and maximum tumor diameter had been assessed macroscopically in most tumors (fig. 1). If not indicated, the local stage according to the TNM classification was reconstructed from the tumor extension in the autopsy report. The macroscopic growth pattern was classified into the following categories: infiltrative, expansive, diffuse and mixed infiltrative/expansive, according to Kojiro [10]. 
Clinical Data. For each case, information about the cause of the death, age at death and gender as well as the underlying liver disease were obtained from the original autopsy report, and the clinical and histological findings were integrated. In cases where no underlying liver disease was described in the autopsy report, additional clinical data archives were searched. If $>1$ underlying liver disease was specified, all were noted. When all data clearly indicated that all known underlying diseases had been excluded, the underlying liver disease was considered absent. In all other cases, where insufficient data were available, the underlying liver diseases were marked as unknown.

Morphological Features. All specimens were taken from formalin-fixed and paraffin-embedded tissue. At least one block containing liver histology was reviewed. We evaluated the neoplastic and non-neoplastic liver tissue of the same patient.

For evaluation, the following stains, at least, were required: hematoxylin and eosin and chromotrope-aniline blue or Sirius red. In most cases, additional stains such as PAS-diastase, Prussian blue and Orcein were also evaluated. Processing was performed using the routine methods of our institution. The diagnosis of a1antitrypsin deficiency relies on postmortem PAS-diastase staining and immunohistochemistry.

The non-neoplastic liver tissue was reviewed to determine fibrosis and inflammatory activity according to the METAVIR scoring system, regardless of a viral hepatitis infection being present or not [11]. METAVIR stage F0/F1 was considered as non-fibrotic or minimally fibrotic, F2 as moderate and F3/F4 as advanced/cirrhotic.

The presence of steatosis and/or steatohepatitis was assessed histologically and quantified according to established criteria [12].

The neoplastic lesions were cytologically graded according to Edmondson and Steiner [13]. The tumors were classified based on their architecture and cytological features according to the World Health Organization's Classification of Tumours of the Digestive System [14] and related publications $[15,16]$. Histomorphological appearance was classified into the following growth patterns: trabecular, pseudoglandular, compact (solid), scirrhous, sarcomatous and fibrolamellar [17].

When $>1$ growth pattern was present and the less prominent occupied at least $5 \%$ of the evaluated tumor mass, the lesion was classified as mixed. In cases of a mixed tumor pattern, each growth pattern was noted. Furthermore, cytological subtypes of the examined tumor area such as clear cell, giant cell, steato-HCC and stem cell-like were noted [17].

The presence of macro- and microvessel infiltration was also noted. This information was gathered from the autopsy report to ensure that macroscopic findings were included but also histologically reevaluated to include microinvasion.

\section{9-1983 Collective}

To compare metastatic patterns, liver fibrosis and underlying hepatic disease, we also reviewed the autopsy reports for 19691983. Data were available from the written autopsy reports, but slides and paraffin blocks were not available. Due to missing data, no other variables could be compared between the 2 groups in this collective. Out of 29,000 cases, we found 240 patients with HCC. The reports were thoroughly studied and location of metastasis, extent of liver fibrosis and underlying liver disease were noted. Paraffin-embedded material from these patients was no longer available and therefore could not be reevaluated. Data for 1984-1987 were not available and are therefore not included in this study.

Features of 398 Autopsy Cases of HCC

\section{Statistical Analysis}

Statistical analysis was performed using SPSS v21. Continuous data are expressed as mean value with standard deviation. Comparisons between 2 groups were made using column proportions tests (z-tests) or column mean tests (t-tests). $\mathrm{p}<0.05$ was considered significant, with $\mathrm{p}$ values being adjusted using the Bonferroni method for multiple comparisons.

\section{Results}

\section{Patient Characteristics}

In all of the cases, the predominant underlying liver disease was due to alcohol consumption. In a gender- and collective-dependent analysis, significantly more males consumed alcohol during both time periods. In the 19691983 collective, significantly more females had viral hepatitis as the underlying liver disease. However, this difference is probably related to the lack at that time of a serological test for the hepatitis $C$ virus. Table 1 summarizes the complete distribution of the underlying liver diseases in both collectives. Interestingly, the non-fibrotic or minimally fibrotic patients showed a history of alcohol consumption in $28.5 \%$ of cases and a hepatitis B virus infection in $14.3 \%$ of cases, while $57.2 \%$ had no underlying liver disease. Furthermore, a total of 18 patients showed $>1$ underlying liver disease; the combination of alcohol consumption and any viral hepatitis was the most frequent (83\%), followed by a1-antitrypsin deficiency and any viral hepatitis (11\%). There was also a single case of alcohol consumption combined with hemochromatosis. Although excess alcohol consumption was the most common cause of the underlying liver disease, our data show no statistically significant risk increase for HCC development due to alcohol in the absence of advanced fibrosis, as most of the cases with minimal fibrosis had no known underlying liver disease. Similarly, a1-antitrypsin deficiency was not present in the cases with absent to mild fibrosis (table 2).

Two patients were excluded from the more recent collective due to the liver tumor being histologically reclassified as cholangiocellular carcinoma or mixed cholangio-HCC.

\section{Tumor Characteristics}

The localization of the HCC was bilobal in $47.3 \%$ of all cases, followed by the right lobe with $34.8 \%$ and the left lobe with $17.9 \%$. No correlation with the underlying liver disease and tumor localization was present.

Trabecular growth was the predominant histologic pattern, followed by mixed- and solid-growth pattern. Of 
Table 2. Measurement of fibrosis in nonneoplastic liver tissue

\begin{tabular}{llllrr}
\hline & \multicolumn{5}{l}{ Fibrosis $^{1}$} \\
& F0 & F1 & F2 & F3 & F4 \\
\hline Absent & 0 & 0 & 3 & 0 & 0 \\
Alcohol & 0 & 5 & 6 & 17 & 170 \\
Viral hepatitis & 0 & 2 & 3 & 3 & 92 \\
NASH/NAFLD & 0 & 0 & 0 & 0 & 0 \\
Hemochromatosis & 0 & 0 & 0 & 0 & 6 \\
PBC/PSC & 0 & 0 & 0 & 2 & 1 \\
a1-antitrypsin deficiency & 0 & 0 & 1 & 0 & 3 \\
Unknown & 3 & 8 & 9 & 10 & 42 \\
\hline
\end{tabular}

NASH/NAFLD = Non-alcoholic steatohepatitis/non-alcoholic fatty liver disease; PBC/PSC = primary biliary cirrhosis/primary sclerosing cholangitis.

${ }^{1}$ Measured according to the METAVIR scoring system.

Table 3. Characteristics of tumors

$1988-2012$

$\begin{array}{lr}\text { Grading }^{1} & \\ \text { Grade } 1 & 0.6 \\ \text { Grade } 2 & 16.5 \\ \text { Grade } 3 & 51.9 \\ \text { Grade } 4 & 29.1 \\ \text { Not evaluable } & 1.9 \\ \text { Growth pattern } & \\ \text { Trabecular } & 72.7 \\ \text { Pseudoglandular } & 13.6 \\ \text { Compact/solid } & 23.4 \\ \text { Scirrhous } & 1.9 \\ \text { Sarcomatous } & 2.6 \\ \text { Fibrolamellar } & 1.9 \\ \text { Cytological subtype } & \\ \text { Clear cell } & 44.4 \\ \text { Giant cell } & 36.1 \\ \text { Steato-HCC } & 22.2 \\ \text { Stem cell-like } & 2.8 \\ \text { Presence of vessel infiltration } & 67.5\end{array}$

Values denote the percentage of patients.

${ }^{1}$ According to Edmondson and Steiner [13].

the cases with mixed-growth pattern, a combination of trabecular and pseudoglandular was seen most often, followed by trabecular and solid.

Cytological variants were seen in $24 \%$ of cases. The clear cell variant was seen in $44.4 \%$ of these cases, followed by the giant cell variant in $36.1 \%$.
Regarding the tumor grading according to Edmondson and Steiner [13], 51.9\% of the tumors were classified as grade 3 (table 3 ). As expected, cytological variants were mainly reported in grade 3/4 tumors. Furthermore, there was no significant association between the presence of steatosis in the non-neoplastic liver and steato-HCC variant, but $5 / 6$ cases with this rare variant occurred in patients with alcohol consumption causing the underlying liver disease.

Macroscopic and/or microscopic angioinvasive growth was observed in $67.5 \%$.

\section{Metastatic Patterns}

Extrahepatic metastasis was found in 156 of 398 HCCs. The incidence of extrahepatic metastases was not significantly different in HCC without cirrhosis and HCC with advanced fibrosis/cirrhosis.

No significant difference was observed in the occurrence of regional nodal metastasis and distant metastasis between the 2 collectives, i.e. 1969-83/1988-2012. Nodal metastases occurred in $17.1 \% / 12.1 \%$ of the cases and distant metastases were seen in $38.6 \% / 39.6 \%$ of the patients, respectively (fig. 2).

In the patients with metastasis, the primary location of the metastasis was the lungs $(61 \% / 73 \%)$. The adrenals were affected in $16 \% / 18 \%$. Spine and bones were affected in $30 \% / 18 \%$ of patients. No significant changes could be observed in the distribution of distant metastasis between 1969-1983 and 1988-2012 (table 4; fig. 2).

\section{Concurrent Causes of Death}

The most frequent concurrent causes of death among all autopsies were cardiovascular including myocardial infarction, thromboembolism of the pulmonary artery and heart failure (table 5). Hypovolemia included gastrointestinal bleeding as well as other sites of bleeding, and was more frequently observed in advanced-stage fibrosis. Tumor rupture was classified into the group of other causes of death.

\section{Discussion}

HCC was detected in 398 of our 44,104 patients that underwent autopsy procedures performed between 1969 and 1983 and between 1988 and 2012 with an overall prevalence of $0.9 \%$. This is less frequent than in previous reporting studies with a frequency of between 1.5 and $6.08 \%[9,18]$. This difference between different autopsy studies in the prevalence of HCC can be attributed to sev- 


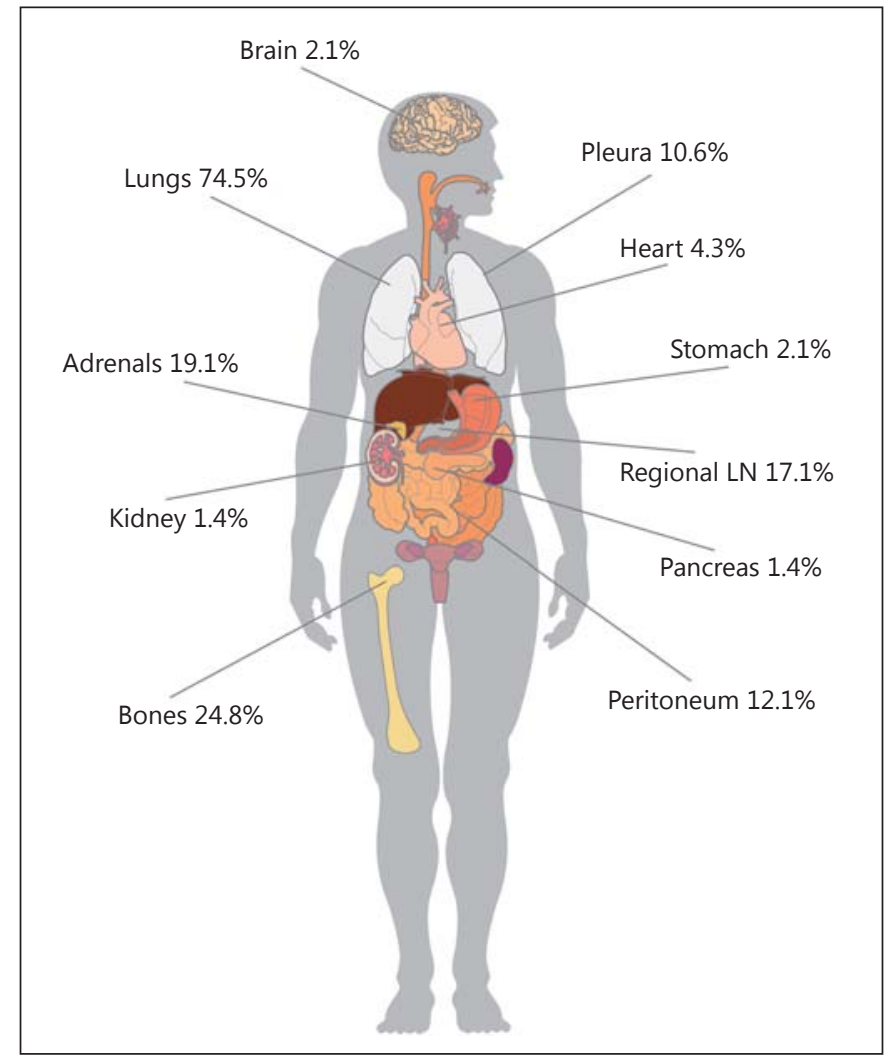

Fig. 2. The schematic of the metastatic patterns summarized for both collectives. $\mathrm{LN}=$ Lymph node.

eral factors including the different geographical incidence of risk factors like viral hepatitis and alcohol intake, different patient populations and particularly to the different methods of tissue examination and processing. Interestingly, in most of the $\mathrm{HCC}$ cases reported by $\mathrm{Na}$ kashima et al. [9], postmortem arteriography and portography were carried out with the injection of colored gelatin solutions containing ultrafine barium sulfate followed by fixation and slicing for soft X-ray imaging. The lower prevalence of HCC in our study could therefore also be due to the fact that only liver nodules macroscopically suspicious for liver cancer were histologically analyzed. This suggests that a number of microscopic carcinomas could be undetected in this series.

As expected, hematogeneous metastases are much more common than lymphatic ones, occurring in 39.2 and $14.1 \%$ of patients, respectively. The lung was by far the most frequent site of metastasis, i.e. in $75 \%$ of the cases, with an incidence higher than previously reported in other series, i.e. approximately $50 \%[9,19]$. As to the other sites of hematogenous metastasis, the lungs were fol-
Table 4. Metastatic patterns separated for both collectives

\begin{tabular}{lcll}
\hline & $1988-2012$ & $1969-1983$ & p value \\
\hline Lungs & 66.1 & 80 & n.s. \\
Adrenals & 17.9 & 20 & n.s. \\
Spine/bone & 32.1 & 20 & n.s. \\
Pleura & 8.9 & 11.8 & n.s. \\
Peritoneum & 12.5 & 11.8 & n.s. \\
Heart & 7.1 & 2.4 & n.s. \\
Brain & 1.8 & 2.4 & n.s. \\
Stomach & 1.8 & 2.4 & n.s. \\
Kidneys & 1.8 & 1.2 & n.s. \\
Pancreas & 1.8 & 1.2 & n.s. \\
\hline
\end{tabular}

Values denote the percentage of patients. n.s. = Not significant.

Table 5. Cause of death

\begin{tabular}{lccl}
\hline & F0-F2 & F3/F4 & p value \\
\hline Hypovolemia & 10.00 & 25.80 & $<0.05$ \\
Pneumonia & 27.50 & 21.40 & n.s. \\
Cardiovascular & 45 & 36.2 & n.s. \\
Liver failure & 2.5 & 8.7 & n.s. \\
Other & 15 & 9 & n.s. \\
\hline
\end{tabular}

Values denote the percentage of patients. n.s. = Not significant.

lowed in frequency by bone, adrenal glands, brain and kidneys. Interestingly, no significant difference was observed in the occurrence of regional nodal metastasis and distant metastasis between the 2 collectives. We are aware that, due to the incompleteness of treatment data in most cases on our database, it is not possible to draw any definitive statement about the role of the different therapeutic approaches developed in the last 30 years for HCC and cirrhosis treatment. Nevertheless, the results of our study could indicate that such different treatments modalities have not substantially changed (impacted on) the metastatic patterns of HCC in our autoptic HCC cohort during the last decades.

Moreover, the strong association of tumor grade and tumor diameter with metastasis underlines the role of these parameters as prognostic factors in liver cancer (table 6). Due to the histological heterogeneity of these tumors, tumor grade, especially in biopsy specimens, may not reflect the true grade of the tumor. However, recent literature data seem to demonstrate the predictive value of tumor grade in needle liver biopsies [20,21]. 
Table 6. Measurement of metastasis

\begin{tabular}{lcc}
\hline & \multicolumn{2}{l}{ Distant metastasis } \\
\cline { 2 - 3 } & M0 & M1 \\
\hline Tumor diameter & & \\
$0-2 \mathrm{~cm}$ & 51 & 4 \\
$2.1-5 \mathrm{~cm}$ & 63 & 9 \\
$5.1-10 \mathrm{~cm}$ & 34 & 33 \\
$>10 \mathrm{~cm}$ & 19 & 42 \\
Grade & & \\
1 & 1 & 0 \\
2 & 25 & 1 \\
3 & 47 & 35 \\
4 & 21 & 25 \\
\hline
\end{tabular}

Values denote the percentage of patients.

${ }^{1}$ According to Edmondson and Steiner [13].

One of the most striking results of our study was a clear-cut increase of HCC without or with only slight fibrosis (F0-F2) detected in the HCC collective of 19882012 in comparison with the HCC cohort of 1969-1982; in our survey, they represent $8 \%$ of all HCC. The etiologic factors of HCC in the non-fibrotic liver as well as the reasons of their increase as a percentage are unclear. However, they represent an important subtype of HCC because the mechanisms involved in hepatocarcinogenesis are different from those observed when extensive liver fibrosis is present. We cannot ignore that at least some of the HCCs arising in livers without significant fibrosis could represent a malignant transformation of hepatocellular adenoma (HCA), although due to the fact that this is a retrospective study, some small lesions may not have been examined, and only 1 HCA case was detected. Albeit still rare, Chang et al. [22] show that HCAs are likely increasing in recent years. This increase is due not only to a better recognition of the lesion but also to life-style changes like the consumption of oral contraceptives/anabolic steroids, with a trend towards an increase of HCA being reported in men. Moreover, it has been proposed that steatosis and several factors associated with metabolic syndrome, such as obesity, diabetes and insulin resistance may predispose to HCC in patients without cirrhosis $[23,24]$. In our cohort, a1-antitrypsin deficiency was, in all instances, associated with moderate to severe liver fibrosis, and can therefore not be considered a risk factor for HCC in a non-fibrotic liver. Due to the advanced disease, there was bilobar HCC involvement of the liver in most of the cases and no significant association between the underlying liver diseases and the tumor localization in the liver could be made.

In general, data based on autopsies may be not adequate for epidemiological inferences due to the selection of patients, change in the interest of the pathologist, etc. As in many other countries, the general autopsy rate has continuously declined, making temporal comparisons difficult [25]. Nevertheless, this work has strengths. It is based on a very large database and provides unique information on the autopsy pathology of HCC in a relatively low-incidence country, with a long time frame (19692012) and a large cohort of autopsies $(44,104)$.

\section{Disclosure Statement}

The authors declare no conflict of interest.

\section{References}

1 El-Serag HB: Epidemiology of hepatocellular carcinoma in USA. Hepatol Res 2007;37(suppl 2):S88-S94

2 Ferlay J, Shin HR, Bray F, et al: Estimates of worldwide burden of cancer in 2008: GLOBOCAN 2008. Int J Cancer 2010;127:28932917.

3 Dyson J, Jaques B, Chattopadyhay D, et al: Hepatocellular cancer: the impact of obesity, type 2 diabetes and a multidisciplinary team. J Hepatol 2014;60:110-117.

4 Forner A, Llovet JM, Bruix J: Hepatocellular carcinoma. Lancet 2012;379:1245-1255.
5 El-Serag HB, Rudolph KL: Hepatocellular carcinoma: epidemiology and molecular carcinogenesis. Gastroenterology 2007; 132: 2557-2576.

6 Han ZG: Functional genomic studies: insights into the pathogenesis of liver cancer. Ann Rev Genom Hum Genet 2012;13:171-205.

7 Bosetti C, Levi F, Boffetta P, et al: Trends in mortality from hepatocellular carcinoma in Europe, 1980-2004. Hepatology 2008;48: 137-145.

8 Giarelli L, Melato M, Zanconati F, et al: Primary liver cancer in non-cirrhotic liver. Epidemiological study based on autopsies performed in Trieste, Italy and Kurume, Japan. J Gastroenterol Hepatol 1991;6:278-282.
9 Nakashima T, Okuda K, Kojiro M, et al: Pathology of hepatocellular carcinoma in Japan. 232 Consecutive cases autopsied in ten years. Cancer 1983;51:863-877.

10 Kojiro M: Pathology of Hepatocellular Carcinoma. Malden, Blackwell, 2006.

11 Lefkowitch JH: Liver biopsy assessment in chronic hepatitis. Arch Med Res 2007;38: 634-643.

12 Brunt EM: Nonalcoholic steatohepatitis: definition and pathology. Semin Liver Dis 2001; 21:3-16.

13 Edmondson HA, Steiner PE: Primary carcinoma of the liver. A study of 100 cases among 48,900 necropsies. Cancer 1954;7:462-503. 
14 Bosman FT: WHO Classification of Tumours of the Digestive System, ed 4. Lyon, WHO International Agency for Research on Cancer, 2010, p 417

15 MacSween RNM, Burt AD, Portmann B, et al: MacSween's Pathology of the Liver. Edinburgh, Churchill Livingstone, 2011.

16 Goodman ZD: Neoplasms of the liver. Mod Pathol 2007;20(suppl 1):S49-S60.

17 Schlageter M, Terracciano LM, D'Angelo S, et al: Histopathology of hepatocellular carcinoma. World J Gastroenterol 2014;20:1595515964.

18 Tiribelli C, Melato M, Croce LS, et al: Prevalence of hepatocellular carcinoma and relation to cirrhosis: comparison of two different cities of the world - Trieste, Italy, and Chiba, Japan. Hepatology 1989;10:998-1002.

19 Ishak KG Goodman ZD, Stocker JT: Tumors of the Liver and Intrahepatic Bile Ducts. Washington, Armed Forces Institute of Pathology, 1999.

20 Caturelli E, Solmi L, Anti M, et al: Ultrasound guided fine needle biopsy of early hepatocellular carcinoma complicating liver cirrhosis: a multicentre study. Gut 2004;53:1356-1362.

21 Colecchia A, Scaioli E, Montrone L, et al: Preoperative liver biopsy in cirrhotic patients with early hepatocellular carcinoma represents a safe and accurate diagnostic tool for tumour grading assessment. J Hepatol 2011; 54:300-305.
22 Chang CY, Hernandez-Prera JC, Roayaie S, et al: Changing epidemiology of hepatocellular adenoma in the United States: review of the literature. Int J Hepatol 2013;2013:604860.

23 Caldwell SH, Crespo DM, Kang HS, et al: Obesity and hepatocellular carcinoma. Gastroenterology 2004;127(5 suppl 1):S97-S103.

24 Paradis V, Zalinski S, Chelbi E, et al: Hepatocellular carcinomas in patients with metabolic syndrome often develop without significant liver fibrosis: a pathological analysis. Hepatology 2009;49:851-859.

25 Lugli A, Anabitarte M, Beer JH: Effect of simple interventions on necropsy rate when active informed consent is required. Lancet 1999;354:1391. 